\title{
NUTRITIONAL STATUS AND ITS ASSOCIATED FACTORS AMONG UNDER FIVE CHILDREN IN BHIMTAR OF SINDHUPALCHOWK DISTRICT NEPAL
}

\author{
Gautam $\mathrm{J}^{1^{*}}$, Acharya $\mathrm{R}^{2}$
}

\begin{abstract}
Affiliation
1. Staff Nurse, Tribhuwan University Teaching Hospital.

2. Matron, Chirayu National Hospital and Medical Institute ${ }^{2}$
\end{abstract}

\section{ARTICLE INFO}

Received : 08 February, 2019
Accepted $: 13$ December, 2019
Published : 31 December, 2019

(C) Authors retain copyright and grant the journal right of first publication with the work simultaneously licensed under Creative Commons Attribution License CC - BY 4.0 that allows others to share the work with an acknowledgment of the work's authorship and initial publication in this journal.

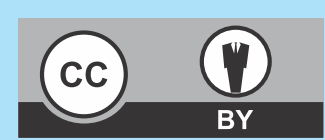

ORA 128

DOI: http://dx.doi.org/10.3126/bjhs.v4i3.27010

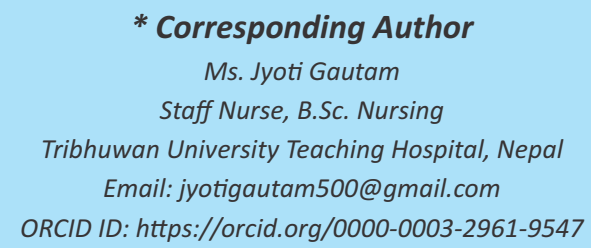

\section{Citation}

Gautam J, Acharya R. Nutritional Status and its Associated Factors among under Five Children in Bhimtar of Sindhupalchowk District Nepal. BJHS 2019;4(3)10: 771-776.

\section{ABSTRACT}

\section{Introduction}

The nutritional status of an individual is often the result of many inter-related factors. It is a major public health problem in most of the developing countries and occurs prominently among under-five children. Nepal is also the one of the developing country where the prevalence of malnutrition is high.

\section{Objective}

This study aimed to assess the nutritional status and its associated factors among under-five children.

\section{Methodology}

A descriptive cross-sectional study was conducted in Bhimtar Village Development Committee of Sindhupalchowk. The study conducted in Jan 272017 to Feb 82017 with the sample 115 , self-constructed semi-structured interview schedule was used for data collection and nutritional status was assessed using anthropometric measurements. Descriptive (Frequency, Percentage, Mean, Standard Deviation) and inferential statistic (chi-square test) were used for data analysis.

\section{Results}

Among total children $47 \%$ were stunted, $30.4 \%$ were underweight and $11.3 \%$ were wasted. Age of child, frequency of antenatal visit and age of mother at child birth had significant association with nutritional status of children.

\section{Conclusion}

The study concludes that significant proportion of underfive children in Sindhupalchowk district were malnourished with stunting and wasting being pre-dominant form of malnutrition. Occurrence of malnutrition was slightly higher among male compared to female children. Child's age, mother's age during child birth and completion of atleast four antenatal visits during pregnancy were found to be significantly associated with child's nutritional status.

\section{KEYWORD}

Nutritional Status, Malnutrition, Under-five Children 


\section{INTRODUCTION}

Nutritional status is a sensitive indicator of community health and nutrition. Nutritional deficiencies raise various morbidities, which in turn, may lead to increased mortality. Malnutrition is a major underlying cause of child morbidity and mortality in developing countries. It affects the child's cognitive and physical development. Globally, the prevalence of stunting and wasting was estimated to be $25 \%$ and $5 \%$ respectively in 2013; where half of all stunted children lived in Asia. ${ }^{1}$ In Nepal $41 \%$ of the under-five children were stunted in 2011where children in rural area were more likely to be stunted than in urban area. ${ }^{2}$

Despite a there is a decline in reduction of maternal child under-nutrition, Nepal still faces high chronic as well as acute under-nutrition in children. ${ }^{3}$ Thus, there is need to assess the nutritional status of under-five children. The objective of this study was to assess the nutritional status and its associating factors among under-five children in Bhimtar VDC of Sindhupalchowk District.

\section{METHODOLOGY}

A descriptive cross sectional design was used for this study. The study was conducted at Bhimtar VDC of Sindhupalchowk District. Sindhupalchowk is one of the most earthquake affected area of Nepal by the Natural disaster in 2015. Children aged 6-59 months old and their mothers who were willing to participate in the study were included in this study. Cluster sampling method was used for sampling. Each ward of the VDC was considered as cluster. Six clusters (ward 1,2,5,6,7,8) were selected by lottery method. Thereafter, under-five children from selected cluster were selected purposively to meet the required sample size of 115 . Sample size was calculated according to

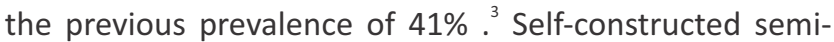
structured interview schedule was used for data collection. Interview schedule was designed to collect data about child information and family practices, feeding practices, hand hygiene and environmental sanitation, immunization, health problem and anthropometric measurements. Stadiometer with movable head piece was used for measurement of height and length, PROFI-med for weight and Non-stretchable Shakir's tape were used to measure MUAC of children. Pre-testing was conducted in $10 \%$ of sample in Tukucha VDC of Kavre District. Validity of tool was ascertained by literature review and subject expert and necessary modifications was done. CDC WHO standard tool was used to assess nutritional status. Data was analysed using descriptive statistics, chi-square and Fischer's Exacttest.

\section{Ethical Considerations}

Written permission was obtained from authority of institutional review sub-committee of Nepal Medical
College and VDC secretary of BhimtarVDC. Informed consent was taken from all participants before data collection. Confidentiality was maintained throughout the study.

\section{RESULTS}

Data was collected from 115 respondents; out of which half (50.4\%) of the respondents were male and one-fourth $(25.2 \%)$ belonged to the age group $13-24$ months. More than half (58.3\%) belonged to nuclear family with $79.1 \%$ of the mother belonging to the age group 20-35 years. Less than $20 \%$ of the parents had education above higher secondary and majority (72.2\%) of them were above, the poverty line. (Table 1). Majority (73.9\%) of the respondent were delivered at health institutions while mean birth weight was $2.7 \mathrm{Kg}$ and birth order being $1^{\text {st }}$ or $2^{\text {nd }}$ in most (80.9\%) of the cases. (Table 2 )

Most (93\%) of the respondents were breastfed within half an hour of birth along with colostrums. Pre-lacteal feed was avoided in $96.5 \%$ of the cases and exclusive breastfeeding was done for six months in majority of cases. Less than $20 \%$ of the respondents had suffered from ARI (17.4\%) and diarrhea (16.5\%) 1 month prior to data collection. (Table 2 )

\begin{tabular}{|c|c|c|c|}
\hline Variables & Frequency & Percent & Remarks \\
\hline $\begin{array}{l}\text { Age of child in months } \\
6-12 \\
13-24 \\
25-36 \\
37-48 \\
49-59\end{array}$ & $\begin{array}{l}21 \\
29 \\
20 \\
23 \\
22\end{array}$ & $\begin{array}{l}18.3 \\
25.2 \\
17.4 \\
20.0 \\
19.1\end{array}$ & $\begin{array}{l}\text { Mean } \pm \text { S.D } \\
=31.03 \pm 16.733 \\
\text { Range (6.59) }\end{array}$ \\
\hline $\begin{array}{l}\text { Sex of child } \\
\text { Male } \\
\text { Female }\end{array}$ & $\begin{array}{l}58 \\
57\end{array}$ & $\begin{array}{l}50.4 \\
49.6\end{array}$ & \\
\hline $\begin{array}{l}\text { Ethnicity } \\
\text { Disadvantaged } \\
\text { Janajatis } \\
\text { Upper caste }\end{array}$ & $\begin{array}{c}103 \\
12\end{array}$ & $\begin{array}{l}89.6 \\
10.4\end{array}$ & \\
\hline $\begin{array}{l}\text { Type of family } \\
\text { Nuclear } \\
\text { Joint }\end{array}$ & $\begin{array}{l}67 \\
48\end{array}$ & $\begin{array}{l}58.3 \\
41.7\end{array}$ & \\
\hline $\begin{array}{l}\text { Family size } \\
<5 \\
5-10 \\
>10\end{array}$ & $\begin{array}{c}55 \\
51 \\
8\end{array}$ & $\begin{array}{c}47.8 \\
44.3 \\
7.0\end{array}$ & $\begin{array}{l}\text { Mean } \pm \\
S . D=5.61 \pm 2.519 \\
(\min , \max )=(3,13)\end{array}$ \\
\hline $\begin{array}{l}\text { Age of mother's at } \\
\text { child birth in years } \\
<20 \\
20-35 \\
>35\end{array}$ & $\begin{array}{c}20 \\
91 \\
4\end{array}$ & $\begin{array}{c}17.4 \\
79.1 \\
3.5\end{array}$ & $\begin{array}{l}\text { Mean } \pm \\
\text { S.D }=23.52 \pm 5.148 \\
(\min , \max ) \\
=(15,46)\end{array}$ \\
\hline $\begin{array}{l}\text { Religion } \\
\text { Hindu } \\
\text { Buddhist }\end{array}$ & $\begin{array}{c}114 \\
1\end{array}$ & $\begin{array}{c}99.1 \\
0.9\end{array}$ & \\
\hline $\begin{array}{l}\text { Socioeconomic status } \\
\text { Below poverty line } \\
\text { Above poverty line }\end{array}$ & $\begin{array}{l}32 \\
83\end{array}$ & $\begin{array}{l}27.8 \\
72.2\end{array}$ & \\
\hline
\end{tabular}


Table 2: Birth History of the Child and feeding Practices $(\mathrm{n}=115)$

\begin{tabular}{|c|c|c|c|}
\hline Variables & Frequency & Percent & Remarks \\
\hline $\begin{array}{l}\text { Place of child delivery } \\
\text { Health post } \\
\text { Hospital } \\
\text { Home delivery }\end{array}$ & $\begin{array}{c}85 \\
21 \\
9\end{array}$ & $\begin{array}{c}73.9 \\
18.3 \\
7.8 \\
\end{array}$ & \\
\hline $\begin{array}{l}\text { Four ANC visit } \\
\text { during pregnancy } \\
\text { Completed } \\
\text { Not completed }\end{array}$ & $\begin{array}{c}103 \\
12\end{array}$ & $\begin{array}{l}89.6 \\
10.4\end{array}$ & \\
\hline $\begin{array}{l}\text { Birth weight of } \\
\text { child in grams(n=108) } \\
<2500 \\
2500-3500 \\
>3500\end{array}$ & $\begin{array}{c}17 \\
88 \\
3\end{array}$ & $\begin{array}{c}15.7 \\
81.5 \\
2.8\end{array}$ & $\begin{array}{l}\text { Mean } \pm S . D= \\
2787.27 \pm 469.76 \\
(\min , \max ) \\
=(1900,4100)\end{array}$ \\
\hline $\begin{array}{l}\text { Birth order of child } \\
1^{\text {st }}-2^{\text {nd }} \\
3^{\text {rd }}-4^{\text {th }} \\
5^{\text {th }}-6^{\text {th }}\end{array}$ & $\begin{array}{c}93 \\
16 \\
6\end{array}$ & $\begin{array}{c}80.9 \\
13.9 \\
5.2 \\
\end{array}$ & $\begin{array}{l}\text { Mean } \pm \text { S.D } \\
=1.86 \pm 1.146 \\
(\min , \max ) \\
=(1,6)\end{array}$ \\
\hline $\begin{array}{l}\text { No of children } \\
\text { of mother's } \\
\text { Up to } 2 \\
3 \text { to } 4 \\
\text { More than } 5\end{array}$ & $\begin{array}{c}90 \\
20 \\
5\end{array}$ & $\begin{array}{c}78.3 \\
17.4 \\
4.3 \\
\end{array}$ & $\begin{array}{l}\text { Mean } \pm \text { S.D } \\
=1.95 \pm 1.146 \\
(\min , \max ) \\
=(1,6)\end{array}$ \\
\hline $\begin{array}{l}\text { Initiation of breast } \\
\text { feeding } \\
\text { Immediately after a } \\
\text { birth/within a half } \\
\text { hour } \\
\text { After half an hour to } \\
\text { within a week }\end{array}$ & 107 & $\begin{array}{l}93 \\
7\end{array}$ & \\
\hline $\begin{array}{l}\text { Colostrum feeding } \\
\text { Yes } \\
\text { No }\end{array}$ & $\begin{array}{c}113 \\
2\end{array}$ & $\begin{array}{c}98.3 \\
1.7\end{array}$ & \\
\hline $\begin{array}{l}\text { Pre-lacteal feeding } \\
\text { Yes } \\
\text { No }\end{array}$ & $\begin{array}{c}4 \\
111\end{array}$ & $\begin{array}{c}3.5 \\
96.5\end{array}$ & \\
\hline $\begin{array}{l}\text { Exclusive } \\
\text { breast feeding } \\
<6 \text { months } \\
\geq 6 \text { months }\end{array}$ & $\begin{array}{l}31 \\
84\end{array}$ & $\begin{array}{l}27 \\
73 \\
\end{array}$ & \\
\hline $\begin{array}{l}\text { Timing of weaning } \\
<6 \text { months } \\
\geq 6 \text { months }\end{array}$ & $\begin{array}{l}31 \\
84\end{array}$ & $\begin{array}{l}27 \\
73\end{array}$ & \\
\hline $\begin{array}{l}\text { Food introduced } \\
\text { during weaning } \\
\text { Rice } \\
\text { Pudding rice } \\
\text { Porridge }\end{array}$ & $\begin{array}{l}60 \\
36 \\
19\end{array}$ & $\begin{array}{l}52.2 \\
31.3 \\
16.5\end{array}$ & \\
\hline $\begin{array}{l}\text { Preparing food for } \\
\text { child separately or } \\
\text { not }\end{array}$ & 29 & 25.2 & \\
\hline
\end{tabular}

Majority (> 80\%) of the respondent's mother had practice of washing hand before and after meal, after use of toilet and after helping child with latrine. Few (3.5\%) did not have toilet in their household. (Table 3)

Anthropometric measurements revealed that out of 115 respondents $47 \%$ were stunted, $30.4 \%$ were under weight and $11.3 \%$ were wasted. Mid-Upper Arm Circumference revealed that one-fourth (25.2\%) were at risk of malnutrition and $4.3 \%$ had moderate acute malnutrition. (Table 5 )
There was significant association of under weight with age of the child ( $p=0.01)$, age of mother during birth of child $(p=0.03)$ and completion of four ANC visit (0.04). (Table 7) Similarly, significant association was established between wasting and completion of 4 ANC visit (0.03). (Table 8)

\begin{tabular}{|c|c|c|c|}
\hline Variables & Frequency & Percent & Remarks \\
\hline $\begin{array}{l}\text { Practice of washing } \\
\text { child's hand before meal } \\
\text { Yes } \\
\text { No }\end{array}$ & $\begin{array}{l}92 \\
23\end{array}$ & $\begin{array}{l}80 \\
20\end{array}$ & \\
\hline $\begin{array}{l}\text { Practice of washing hands } \\
\text { after using toilet } \\
\text { Always } \\
\text { Sometimes }\end{array}$ & $\begin{array}{c}109 \\
6\end{array}$ & $\begin{array}{c}94.8 \\
5.2\end{array}$ & \\
\hline $\begin{array}{l}\text { Practice of washing hands } \\
\text { before preparing meal } \\
\text { Always } \\
\text { Sometimes }\end{array}$ & $\begin{array}{c}109 \\
6\end{array}$ & $\begin{array}{c}94.8 \\
5.2\end{array}$ & \\
\hline $\begin{array}{l}\text { Practice of washing hands } \\
\text { before feeding child } \\
\text { Always } \\
\text { Sometimes }\end{array}$ & $\begin{array}{c}108 \\
7\end{array}$ & $\begin{array}{c}93.9 \\
6.1 \\
\end{array}$ & \\
\hline $\begin{array}{l}\text { Practice of washing hands } \\
\text { after helping child with } \\
\text { latrine visit } \\
\text { Always } \\
\text { Sometimes }\end{array}$ & $\begin{array}{c}109 \\
6\end{array}$ & $\begin{array}{c}94.8 \\
5.2\end{array}$ & \\
\hline $\begin{array}{l}\text { Garbage disposal method } \\
\text { Digging } \\
\text { Burying } \\
\text { Composting } \\
\text { Throwing randomly }\end{array}$ & $\begin{array}{c}7 \\
34 \\
26 \\
48\end{array}$ & $\begin{array}{c}6.1 \\
29.6 \\
22.6 \\
41.7\end{array}$ & \\
\hline $\begin{array}{l}\text { Presence of toilet facility } \\
\text { Yes } \\
\text { No }\end{array}$ & $\begin{array}{c}111 \\
4\end{array}$ & $\begin{array}{c}96.5 \\
3.5 \\
\end{array}$ & \\
\hline $\begin{array}{l}\text { Source of drinking water } \\
\text { River } \\
\text { Pond } \\
\text { Tap }\end{array}$ & $\begin{array}{c}3 \\
2 \\
110\end{array}$ & $\begin{array}{l}2.6 \\
1.7 \\
95.7 \\
\end{array}$ & \\
\hline $\begin{array}{l}\text { Method of water purification } \\
\text { By filtration } \\
\text { By boiling } \\
\text { Without any purification }\end{array}$ & $\begin{array}{c}12 \\
2 \\
101\end{array}$ & $\begin{array}{c}10.4 \\
1.7 \\
87.8\end{array}$ & \\
\hline
\end{tabular}

Table 4: Health Problems of Child and Health Seeking Behavior $(n=115)$

\begin{tabular}{|l|c|c|}
\hline Variables & Frequency & Percent \\
\hline \hline Suffered from ARI in last & 20 & 17.4 \\
one month & 95 & 82.6 \\
Yes & & \\
No & 19 & 16.5 \\
\hline $\begin{array}{l}\text { Suffered from diarrheal } \\
\text { disease in last one }\end{array}$ & 96 & 83.5 \\
month & & \\
Yes & & \\
No & 19 & 16.5 \\
\hline Place for treatment & 96 & 83.5 \\
\hline Hospital & \\
Health post & & \\
\hline
\end{tabular}




Table 5: Nutritional Status of Children (n=115)
\begin{tabular}{|l|c|c|}
\hline Variables & Frequency & Percent \\
\hline Stunting & & \\
Yes & 54 & 47 \\
No & 61 & 53 \\
\hline Under weight & & \\
Yes & 35 & 30.4 \\
No & 80 & 69.6 \\
\hline Wasting & & \\
Yes & 13 & 11.3 \\
No & 102 & 88.7 \\
\hline MUAC & & \\
$11-12.4$ & 5 & 4.3 \\
\hline $12.5-13.5$ & 29 & 25.2 \\
\hline$>13.5$ & 81 & 70.4 \\
\hline
\end{tabular}

\begin{tabular}{|c|c|c|c|c|c|}
\hline \multirow[t]{2}{*}{ Characteristics } & \multirow[t]{2}{*}{ Category } & \multicolumn{4}{|c|}{ Stunting } \\
\hline & & Yes & No & $x^{2}$ & P valut \\
\hline \multirow{2}{*}{$\begin{array}{l}\text { Age of child in } \\
\text { months }\end{array}$} & $6-24$ & 20 & 23.47 & \multirow{2}{*}{0.03} & \multirow[t]{2}{*}{0.85} \\
\hline & $25-59$ & 26.9 & 29.56 & & \\
\hline \multirow[t]{2}{*}{ Sex } & Male & 24.34 & 26 & \multirow[t]{2}{*}{0.08} & \multirow[t]{2}{*}{0.77} \\
\hline & Female & 22.60 & 27 & & \\
\hline \multirow[t]{2}{*}{ Ethnicity } & $\begin{array}{c}\text { Disadvantaged } \\
\text { Janajatis }\end{array}$ & 44.34 & 45.21 & \multirow[t]{2}{*}{2.59} & \multirow[t]{2}{*}{0.10} \\
\hline & Upper caste & 2.60 & 7.82 & & \\
\hline \multirow{2}{*}{$\begin{array}{l}\text { Birth weight of } \\
\text { child }\end{array}$} & $<2500$ & 7.82 & 6.9 & \multirow[t]{2}{*}{1.21} & \multirow[t]{2}{*}{0.72} \\
\hline & $\geq 2500$ & 38.2 & 40.86 & & \\
\hline \multirow{2}{*}{$\begin{array}{l}\text { Age of mother } \\
\text { at child birth }\end{array}$} & $<20$ & 10.40 & 6.9 & \multirow[t]{2}{*}{1.65} & \multirow[t]{2}{*}{0.19} \\
\hline & $\geq 20$ & 36.52 & 46.08 & & \\
\hline \multirow{2}{*}{$\begin{array}{l}\text { Education level } \\
\text { of mother }\end{array}$} & Illiterate & 0.86 & 6.08 & \multirow[t]{2}{*}{4.09} & \multirow[t]{2}{*}{$0.06^{*}$} \\
\hline & Literate & 46.08 & 46.95 & & \\
\hline \multirow[t]{2}{*}{$\begin{array}{l}\text { Socio-economic } \\
\text { status }\end{array}$} & $\begin{array}{c}\text { Below poverty } \\
\text { line }\end{array}$ & 10.43 & 17.40 & \multirow[t]{2}{*}{1.59} & \multirow[t]{2}{*}{0.20} \\
\hline & $\begin{array}{c}\text { Above poverty } \\
\text { line }\end{array}$ & 36.52 & 35.65 & & \\
\hline \multirow[t]{2}{*}{$\begin{array}{l}\text { Initiation of } \\
\text { breast feeding }\end{array}$} & $\begin{array}{l}\text { Within half } \\
\text { hour }\end{array}$ & 44.34 & 48.70 & \multirow[t]{2}{*}{0.30} & \multirow[t]{2}{*}{$0.72^{*}$} \\
\hline & After half hour & 2.60 & 4.30 & & \\
\hline \multirow{2}{*}{$\begin{array}{l}\text { Exclusive breast } \\
\text { feeding }\end{array}$} & $<6$ months & 11.30 & 15.65 & \multirow[t]{2}{*}{0.43} & \multirow[t]{2}{*}{0.51} \\
\hline & $\geq 6$ months & 35.65 & 37.40 & & \\
\hline \multirow{2}{*}{$\begin{array}{l}4 \text { ANC visit } \\
\text { completion }\end{array}$} & Yes & 43.47 & 46.08 & \multirow[t]{2}{*}{0.99} & \multirow[t]{2}{*}{0.31} \\
\hline & No & 3.47 & 7 & & \\
\hline History of ARI & Present & 11.30 & 6.08 & 3.16 & 0.07 \\
\hline & Absent & 35.65 & 46.95 & & \\
\hline History of & Present & 7.82 & 8.7 & 0.00 & 0.96 \\
\hline $\begin{array}{l}\text { Diarrhoeal } \\
\text { disease in last } \\
1 \text { month }\end{array}$ & Absent & 39.13 & 44.34 & & \\
\hline
\end{tabular}

* Fisher's ExacttestP-value is significant at $\leq 0.05$ levels
Table 7: Association of Underweight with the Characteristics of Children. ( $n=115)$

\begin{tabular}{|c|c|c|c|c|c|}
\hline \multirow[t]{2}{*}{ Characteristics } & \multirow[t]{2}{*}{ Category } & \multicolumn{4}{|c|}{ Underweight } \\
\hline & & Yes & No & $x^{2}$ & P value \\
\hline \multirow{2}{*}{$\begin{array}{l}\text { Age of child in } \\
\text { months }\end{array}$} & $6-24$ & 7.82 & 35.65 & \multirow[t]{2}{*}{6.46} & \multirow[t]{2}{*}{0.01} \\
\hline & $25-59$ & 22.60 & 33.91 & & \\
\hline \multirow[t]{2}{*}{ Sex } & Male & 17.40 & 33.04 & \multirow[t]{2}{*}{0.90} & \multirow[t]{2}{*}{0.34} \\
\hline & Female & 13.04 & 36.52 & & \\
\hline \multirow[t]{2}{*}{ Ethnicity } & $\begin{array}{l}\text { Disadvantag } \\
\text { ed Janajatis }\end{array}$ & 27.82 & 61.73 & \multirow[t]{2}{*}{0.18} & \multirow[t]{2}{*}{$1.0^{*}$} \\
\hline & Upper caste & 2.60 & 7.82 & & \\
\hline \multirow{2}{*}{$\begin{array}{l}\text { Birth weight of } \\
\text { child }\end{array}$} & $<2500$ & 5.21 & 9.56 & \multirow[t]{2}{*}{0.21} & \multirow[t]{2}{*}{0.64} \\
\hline & $\geq 2500$ & 23.47 & 55.65 & & \\
\hline \multirow{2}{*}{$\begin{array}{l}\text { Age of mother at } \\
\text { child birth }\end{array}$} & $<20$ & 8.70 & 8.70 & \multirow[t]{2}{*}{4.37} & \multirow[t]{2}{*}{0.03} \\
\hline & $\geq 20$ & 21.74 & 60.86 & & \\
\hline \multirow{2}{*}{$\begin{array}{l}\text { Education level of } \\
\text { mother }\end{array}$} & Illiterate & 1.74 & 5.21 & \multirow[t]{2}{*}{0.12} & \multirow[t]{2}{*}{$1.0^{*}$} \\
\hline & Literate & 28.70 & 64.34 & & \\
\hline \multirow[t]{2}{*}{$\begin{array}{l}\text { Socio-economic } \\
\text { status }\end{array}$} & $\begin{array}{c}\text { Below } \\
\text { poverty line }\end{array}$ & 8.70 & 19.13 & \multirow[t]{2}{*}{0.01} & \multirow[t]{2}{*}{0.90} \\
\hline & $\begin{array}{c}\text { Above } \\
\text { poverty line }\end{array}$ & 21.74 & 50.43 & & \\
\hline \multirow[t]{2}{*}{$\begin{array}{l}\text { Initiation of } \\
\text { breast feeding }\end{array}$} & $\begin{array}{l}\text { Within half } \\
\text { hour }\end{array}$ & 27.82 & 64.35 & \multirow[t]{2}{*}{0.12} & \multirow[t]{2}{*}{$1.0^{*}$} \\
\hline & $\begin{array}{l}\text { After half } \\
\text { hour }\end{array}$ & 1.74 & 5.21 & & \\
\hline \multirow{2}{*}{$\begin{array}{l}\text { Exclusive breast } \\
\text { feeding }\end{array}$} & $<6$ months & 8.70 & 18.26 & \multirow[t]{2}{*}{0.06} & \multirow[t]{2}{*}{0.79} \\
\hline & $\geq 6$ months & 21.74 & 51.30 & & \\
\hline \multirow{2}{*}{$\begin{array}{l}4 \text { ANC visit } \\
\text { completion }\end{array}$} & Yes & 24.35 & 65.21 & \multirow[t]{2}{*}{4.92} & \multirow[t]{2}{*}{$0.04 *$} \\
\hline & No & 6.08 & 4.34 & & \\
\hline History of ARI in & Present & 5.21 & 12.17 & 0.00 & 0.96 \\
\hline last 1 month & Absent & 25.22 & 57.40 & & \\
\hline History of & Present & 4.34 & 12.17 & 0.18 & 0.66 \\
\hline $\begin{array}{l}\text { Diarrhoeal } \\
\text { disease in last } 1 \\
\text { month }\end{array}$ & Absent & 26.08 & 59.13 & & \\
\hline
\end{tabular}

* Fisher's Exact test $\quad$ P-value is significant at $\leq 0.05$ levels

Table 8: Association of Wasting with the Characteristics of Children

\begin{tabular}{|c|c|c|c|c|c|}
\hline \multirow[t]{2}{*}{ Characteristics } & \multirow[t]{2}{*}{ Category } & \multicolumn{4}{|c|}{ Wasting } \\
\hline & & Yes & No & $x^{2}$ & P value \\
\hline \multirow{2}{*}{$\begin{array}{l}\text { Age of child in } \\
\text { months }\end{array}$} & $6-24$ & 5.21 & 38.26 & \multirow[t]{2}{*}{0.04} & \multirow[t]{2}{*}{0.83} \\
\hline & $25-59$ & 6.08 & 50.43 & & \\
\hline \multirow[t]{2}{*}{ Sex } & Male & 6.08 & 44.34 & \multirow[t]{2}{*}{0.06} & \multirow[t]{2}{*}{0.79} \\
\hline & Female & 5.21 & 44.34 & & \\
\hline \multirow[t]{2}{*}{ Ethnicity } & $\begin{array}{c}\text { Disadvantage } \\
\text { d Janajatis }\end{array}$ & 10.43 & 79.13 & \multirow[t]{2}{*}{0.11} & \multirow[t]{2}{*}{$1.00^{*}$} \\
\hline & Upper caste & 0.86 & 9.56 & & \\
\hline \multirow{2}{*}{$\begin{array}{l}\text { Birth weight } \\
\text { of child }\end{array}$} & $<2500$ & 2.60 & 12.17 & \multirow[t]{2}{*}{0.60} & \multirow[t]{2}{*}{$0.42^{*}$} \\
\hline & $\geq 2500$ & 8.69 & 70.43 & & \\
\hline \multirow{2}{*}{$\begin{array}{l}\text { Age of mother } \\
\text { at child birth }\end{array}$} & $<20$ & 3.47 & 13.91 & \multirow[t]{2}{*}{1.82} & \multirow[t]{2}{*}{$0.23^{*}$} \\
\hline & $\geq 20$ & 7.82 & 74.78 & & \\
\hline \multirow{2}{*}{$\begin{array}{l}\text { Education level } \\
\text { of mother }\end{array}$} & Illiterate & 0.86 & 6.1 & \multirow[t]{2}{*}{0.01} & \multirow[t]{2}{*}{$1.00^{*}$} \\
\hline & Literate & 10.43 & 82.60 & & \\
\hline \multirow[t]{2}{*}{$\begin{array}{l}\text { Socio-economic } \\
\text { status }\end{array}$} & $\begin{array}{c}\text { Below } \\
\text { poverty line }\end{array}$ & 5.21 & 22.60 & \multirow[t]{2}{*}{2.45} & \multirow[t]{2}{*}{$0.18^{*}$} \\
\hline & $\begin{array}{c}\text { Above } \\
\text { poverty line }\end{array}$ & 6.08 & 66.08 & & \\
\hline \multirow[t]{2}{*}{$\begin{array}{l}\text { Initiation of breast } \\
\text { feeding }\end{array}$} & $\begin{array}{l}\text { Within half } \\
\text { hour }\end{array}$ & 11.30 & 81.73 & \multirow[t]{2}{*}{1.09} & \multirow[t]{2}{*}{$0.59 *$} \\
\hline & $\begin{array}{l}\text { After half } \\
\text { hour }\end{array}$ & 0 & 7 & & \\
\hline \multirow{2}{*}{$\begin{array}{l}\text { Exclusive breast } \\
\text { feeding }\end{array}$} & $<6$ months & 3.47 & 23.47 & \multirow[t]{2}{*}{0.10} & \multirow[t]{2}{*}{$0.74^{*}$} \\
\hline & $\geq 6$ months & 7.82 & 65.21 & & \\
\hline \multirow{2}{*}{$\begin{array}{l}4 \text { ANC visit } \\
\text { completion }\end{array}$} & Yes & 7.82 & 81.73 & \multirow[t]{2}{*}{6.48} & \multirow[t]{2}{*}{$0.03^{*}$} \\
\hline & No & 3.47 & 7 & & \\
\hline \multirow{2}{*}{$\begin{array}{l}\text { History of ARI in } \\
\text { last } 1 \text { month }\end{array}$} & Present & 2.6 & 14.78 & 0.33 & 0.69 \\
\hline & Absent & 8.69 & 73.91 & & \\
\hline $\begin{array}{l}\text { History of Diarrhoeal } \\
\text { disease in last } 1 \text { month }\end{array}$ & Present & 0.86 & 15.65 & 0.82 & $0.69 *$ \\
\hline
\end{tabular}

* Fisher's Exact test 


\section{DISCUSSION}

The current study showed that among the total participants, $47 \%$ were stunted, $30.4 \%$ were underweight and $11 \%$ were wasted which is nearly similar to the national finding by the Nepal Demographic Health Survey 2011 which estimated that $41 \%$ of children under five years old were stunted, $29 \%$ were underweight and $11 \%$ were wasted. ${ }^{4}$

Prevalence of stunting (47\%) which is indicator of chronic malnutrition is similar to the result of different studies in different setting. ${ }^{5-9}$ Prevalence of underweight in the current study was $30.4 \%$ which is $7 \%$ less than the results found by the study conducted in Padampur VDC ${ }^{10}$ and Agro-pastoral community of Blue Hora district. ${ }^{8}$ However, prevalence of underweight in the current study is greater than the other national international studies conducted in Dolakha and Kavre districts of Nepal, in Liabela town, Northern Ethiopia. ${ }^{7}$ and in Agro- pastoral community of Blue Hora District. ${ }^{5,7,8}$

Wasting, an indicator of acute malnutrition had prevalence similar to the study conducted among the street children in India. ${ }^{9}$ However, higher prevalence was found in the study conducted in Dolakha, Nepal and Liabela town. ${ }^{5,8}$

\section{Factors associated with Nutritional Status}

The findings of the current study showed that there was significant association of nutritional status with mother's age and ANC visit during pregnancy which is supported by other studies conducted in Nepal. ${ }^{6,11,12}$ The study further demonstrated that there is significant association of nutritional status with age of the child which is supported by the study in Liabela town, Blue Hora district and western Ethiopia. ${ }^{7,8,13}$

There is no any significant association was between nutritional status and morbidity which is supported by the study conducted in Padampur VDC. ${ }^{3}$ However, study in Brazil and Kapilvastu established significant association. ${ }^{12,14}$

This study showed that there is no any significant association of nutritional status with sex, ethnicity, occupation of mother, socio-economic status of the family, birth weight, birth order, birth spacing education level of mother, exclusive breast feeding, feeding practices, family type and size in contrast to other similar studies which showed significant association. $5,7,8,11,12,13,15,16$

\section{CONCLUSION}

The study concludes that significant proportion of underfive children in Sindhupalchowk district is malnourished with stunting and wasting being predominant form of malnutrition. Occurrence of malnutrition was slightly higher among male compared to female children. Child's age, mother's age during the birth of child and their completion of at least 4 antenatal visits during pregnancy were found to be significantly associated with child's nutritional status.

\section{RECOMMENDATIONS}

The findings suggests awareness program to the mother's needs to be strengthened regarding appropriate age of giving birth to the child and importance of ANC visits during pregnancy.

\section{LIMITATION OF THE STUDY}

Nutritional status was assessed using only anthropometric measurements.

Study was limited to the under - five children residing in Bhimtar VDC of SIndhupalchowk district.

\section{ACKNOWLEDGEMENT}

The author would like to acknowledge all participants and their mother who have provided enormous support and cooperation during the study.

\section{CONFLICT OF INTEREST}

There is no any conflict of interest during study.

5. Acharya D, GautamS ,Kaphle H ,Neupane N. Factors Associated with Nutritional Status of Under Five Children in Rupendehi District of Nepal : JHealth Allied Sci2013; 3:56-9. PMID: 272485586

6. HailemariamWoldeTsedeke .Prevalnce of Underweight and its Determinant Factors of Under Two Children in a Rural Area of Western Ethiopia :J Food Sci Quality Man 2014; 31:59-69. PMID: 279923887

7. Santos de AraújoThiago, Oliveira CristieliSérgio de Menezes, Torres Muniz Pascoal , Silva-Nunes ,Mônica da, Cardoso Marly Augusto .Child undernutrition in one of the cities with greater nutritional risk in Brazil: population-based study in the Western Brazilian Amazon REVBRAS EPIDEMIOL JUL-SET 2016; 19: 554-66. DOI: 10.1590/ 19805497201600030007

8. Rijal P, Sharma A, Shrestha S, Upadhyay S. Nutritional assessment of children at Nepal Medical College Teaching Hospital. Health Renaiss 2011;9:184-8. DOI: 10.3126/hren.v9i3.5588 
9. Ruwali D. Nutritional Status of Children Under Five Years of Age and Factors Associated in Padampur VDC, Chitwan:Health Prospect 2011; 10:14-8. DOI: DOI: 10.3126/hprospect.v10i0.5639

10. Bhandari TR, ChhetriM.Nutritional Status of Under Five Year Children and Factors Associated in Kapilvastu District, Nepal:JNut Health Food Sci 2013;1(1):6. DOI: 10.15226/jnhfs.2013.00106

11. Niraula SR et. al. Prevalence and associated risk factors with malnutrition among under-five Nepalese children of Borbote village, Ilam:HealthRenaiss 2013;11;111-18. DOI: 10.3126/hren. v11i2.8217

12. MandefroAsfaw, MekitieWondaferash, Mohammed Taha and LamesaDube.Prevalance of Undernutrition and Associated Factors among Children Aged between Six to Fifty nine months in Blue Hora district ,South Ethiopia:Bio Med Central Public Health 2015;15:41. DOI: 10.1186/s12889-015-1370-9
13. RathodNeelam ,WarbhePriya . Assessment of Nutritional Status of Street Children in Selected Wards of an Urban Area: Inter JInter disciplinary Multidisciplinary Studies, 2014; 1: 136-43

14. Dhungana GP. Nutritional Status of Under 5 Children and Associated Factors of Kuncha Village Development Committee:JChitwan Med Col 2013; 3:38-42.

15. Food and Agriculture Organization of United Nations (FAO). Fact Sheet: Gender and Nutrition. 